\title{
TRATAMIENTO POR DECLORINACIÓN IN SITU DE BIFENILOS POLICLORADOS (PCBS), PARA CONTROL DE RIESGOS DE SALUD DE LOS TRABAJADORES Y EL MEDIO AMBIENTE EN EL SECTOR MINERO DEL DEPARTAMENTO DE PASCO
}

\author{
Jessica Muñoz Hermitaño*
}

\begin{abstract}
RESUMEN
El estudio describe el manejo y la aplicación del tratamiento por declorinación de bifenilos policlorados, o más conocidos por sus siglas en inglés "polychlorinated biphenyls" (PCBs), detectándose en los aceites dieléctricos de los transformadores de tensión antiguos del lugar de estudio. El manejo inicia en la identificación y toma de muestras de las fuentes, considerando cuarenta y dos transformadores para el proyecto, luego se realizó la prueba de descarte, haciendo uso de los kits colorimétricos Clor-N-Oil 50, para detección de PCBs, resultando trece equipos como positivos, estos equipos se volvieron a muestrear y enviar las muestras para su análisis por cromatografía de gases en un laboratorio acreditado, para obtener resultados exactos de PCBs, resultando nueve transformadores con presencia de PCBs superior a $50 \mathrm{ppm}$, a los cuales se aplicó el tratamiento por declorinación, mediante un reactor de 1500 litros, en donde se genera la "reacción química de Wurtz", entre el aceite dieléctrico y el sodio metálico, generándose reacciones de eliminación y sustitución del halógeno cloro presente en el aceite y convirtiéndose en cloruro de sodio, el cual es completamente inerte y no posee propiedades nocivas para la salud de las personas y el medio ambiente.
\end{abstract}

Palabras clave: Bifenilos policlorados (PCBs), tratamiento por declorinación, cloro.

\footnotetext{
* Unidad de Posgrado de la Facultad de Ingeniería Geológica, Minera, Metalúrgica y Geográfica, de la Universidad Nacional Mayor de San Marcos, Av. Venezuela s/n cuadra 24, Lima - Perú. E-mail: jessicazaida1@ gmail.com.

Dirección de la Facultad de Ingeniera Metalúrgica de la Universidad Nacional Mayor de San Marcos.
} 


\title{
TREATMENT FOR DECHLORINATION IN SITU OF POLYCHLORINATED BIPHENYLS (PCBs), FOR CONTROL OF HEALT RISKS FROM THE WORKERS AND THE ENVIRONMENT IN THE MINING SECTOR OF THE PASCO DEPARTMENT
}

\begin{abstract}
The study describes the management and application of the treatment by dechlorination of polychlorinated biphenyls, or better known by its abbreviations (PCBs), detected in the dielectric oils of the old voltage transformers of the place of study. The management begins with the identification and sampling of the sources, considering forty-two transformers for the project, then the discard test was carried out, using the Clor-N-Oil 50 colorimetric kits, to detect PCBs, resulting in thirteen teams as positive, these devices were re-sampled and the samples sent for analysis by gas chromatography in an accredited laboratory, to obtain exact results of PCBs, resulting in nine transformers with PCBs greater than $50 \mathrm{ppm}$, which was applied the treatment by dechlorination, by means of a reactor of 1500 liters, where the "chemical reaction of Wurtz" is generated, between the dielectric oil and the metallic sodium, generating reactions of elimination and substitution of the chlorine halogen present in the oil and becoming sodium chloride, which is completely inert and has no harmful properties for the health of people and the environment ambient.
\end{abstract}

Key words: Polychlorinated biphenyls (PCBs), dechlorination treatment, chlorine.

\section{INTRODUCCIÓN}

Los bifenilos policlorados son productos industriales derivados de la industria petroquímica (hidrocarburos aromáticos clorados) y de los que no se conocen fuentes naturales ${ }^{1}$. Su fórmula general es $\mathrm{C}_{12} \mathrm{H}_{10}$-nCLn (n puede variar entre 1 a 10) (figura 1), su estructura química básicamente está formada de dos anillos bencénicos unidos entre sí dando lugar al bifenilo, que sería la estructura de la molécula base de los PCBs, esta molécula mediante la reacción química en presencia de un catalizador, se puede obtener diferentes grados de cloración ${ }^{2}$. La familia de los PCBs está formada por 209 congéneres, los cuales varían de acuerdo al grado de cloración y la posición de sustitución en los anillos aromáticos ${ }^{3}$.

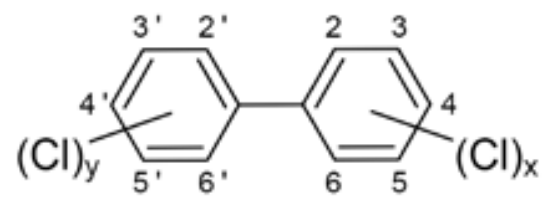

Figura 1. Fórmula general del PCB. Los números 2-6 y 2'-6' representan posibles posiciones del átomo de cloro dentro de cada anillo bencénico. 
Los PCBs han sido aprovechados en numerosos usos industriales a partir del año 1929, empleándose como fluidos aislantes de transformadores y condensadores, plastificantes y fluidos hidráulicos por su característica de resistencia al calor ${ }^{4}$, hasta que la ley para el control de sustancias tóxicas (TSCA) de Estados Unidos y administrada por la Agencia de Protección Ambiental de los Estados Unidos, reguló la introducción de productos químicos nuevos o ya existentes, prohibió su producción de PCBs, debido a su extrema persistencia en el ambiente, su capacidad de bioacumulación y sus efectos adversos sobre la salud humana ${ }^{5}$. Debido a la presencia del cloro, se configuran en compuestos muy estables y resistentes a la degradación química, biológica, mecánica y térmica, tienen una baja presión de vapor y elevados puntos de inflamación; no son hidrolizables ni solubles en agua, pero sí en solventes orgánicos, como aceites y grasas vegetales o sintéticas; características por las cuales son clasificados como compuestos orgánicos persistentes (COPs $)^{6}$.

Con el tiempo se fueron haciendo descubrimientos respecto a los efectos de contaminación que generaban los bifenilos policlorados, como problemas genéticos en aves, concentraciones significantes en lugares lejanos de las plantas productoras y epidemias humanas como las del Yusho (Japón) y Yu-Cheng (Taiwán), por inhalación y contacto de alta concentraciones de PCBs, hasta 1960 en que la comunidad científica mundial reconoció a los PCBs como una amenaza para el ambiente y la salud de las personas, debido a sus efectos nocivos, persistentes y bioacumulables en los tejidos grasos y demás órganos específicos ${ }^{7}$. Existen tres rutas de exposición a través de las cuales los PCB pueden ingresar al organismo: por inhalación por el sistema respiratorio, la ingestión por el sistema digestivo (consumo de alimentos, agua y leche materna proveniente de una madre contaminada) y la absorción a través de la piel durante labores y/o accidentes de trabajo ${ }^{8}$.

Es importante mencionar que en el Perú no se fabricaron estos compuestos, pero la importación de equipos y fluidos fue significativa en muchos sectores del país, siendo el sector minero como uno de los principales usuarios de equipos eléctricos desde hace muchos años. En la actualidad, aún existen empresas mineras del Perú que cuentan con transformadores con contenido de PCBs, siendo que las actividades de intervención a estos equipos tienen una importancia protagónica, ya que los trabajadores corren el riesgo de contacto ya sea por vía respiratoria, por ingesta o por el tacto, que podrían afectar su salud a un corto o largo plazo, también representan un riesgo para el medio ambiente por la liberación voluntaria e involuntaria a los medios receptores (agua, suelo y aire) ${ }^{9}$.

Ante al cumplimiento del tratado de Estocolmo, se idearon muchas tecnologías para el tratamiento de los PCBs, como las tecnologías destructivas, de incineración y hasta de aprovechamiento como es el tratamiento por declorinación ${ }^{10}$. El presente trabajo describe el manejo y la aplicación del tratamiento por declorinación, a los transformadores con contenido de PCBs superior a 50 ppm, desarrollándose por mucho tiempo e iniciando en la identificación de las fuentes con PCBs, luego se realizó la aplicación de descarte con el uso del kit de detección de PCBs Clor-N-Oil 50, para detectar concentraciones de PCB mayor a $50 \mathrm{ppm}$, una vez detectados los equipos positivos con PCBs, estos pasaron por el análisis por cromatografía de gases en un laboratorio acreditado, para obtener valores exactos de PCBs, 
considerando aquellos trasformadores con resultados mayor a $50 \mathrm{ppm}$, para el tratamiento por declorinación. Siendo este tratamiento uno de los métodos más utilizados en otros países, el cual consiste en la reacción entre el fluido dieléctrico y un metal alcalino como en este caso del sodio metálico, el cual reacciona con los átomos de cloro de los PCBs, generando sales de cloruro de sodio y otros productos residuales no halogenados, las sales inorgánicas producidas pueden ser removidas de la fracción orgánica por medio de las filtraciones ${ }^{11}$.

En el Perú, las medidas adoptadas y actividades realizadas tanto por autoridades como por entidades del sector privado han tomado como referencia principal lo estipulado en los Convenios de Basilea y de Estocolmo, los mismos que forman parte de la normatividad nacional al haber sido ratificados ${ }^{12}$. Respecto al marco legal nacional, el día 20/07/2018, mediante la Resolución Ministerial N 683-2018/MINSA, se efectuó la publicación del proyecto de Reglamento Técnico para la Gestión Sanitaria y Ambiental de los bifenilos policlorados, en el portal institucional, el cual, una vez aprobado, fortalecerá la obligación para que aquellos propietarios de equipos con contenido de PCBs realicen una adecuada gestión de los bifenilos policlorados ${ }^{13}$.

\section{PARTE EXPERIMENTAL}

La presente investigación, de acuerdo a las características de las hipótesis formuladas y los objetivos propios de la investigación, ha sido enmarcada dentro del tipo explicativo descriptivo, y el diseño es de investigación experimental.

La metodología planteada tiene por objetivo describir e indicar, a modo específico, todos los pasos seguidos para cumplir con el objetivo principal de este trabajo de investigación, "Tratamiento por declorinación in situ de los PCBs, para control de riesgos de salud de los trabajadores y el medio ambiente en el sector minero del Departamento de Pasco", y poder dejar esta experiencia como un modelo de gestión del manejo de los bifenilos policlorados, para aquellas empresas propietarias de transformadores eléctricos con aceites dieléctricos con PCBs en el Perú.

En la figura 2 se observa el flujograma del desarrollo de la investigación, se describen a continuación cada uno de los procesos, para llegar a la parte final que es la interpretación de los resultados. 


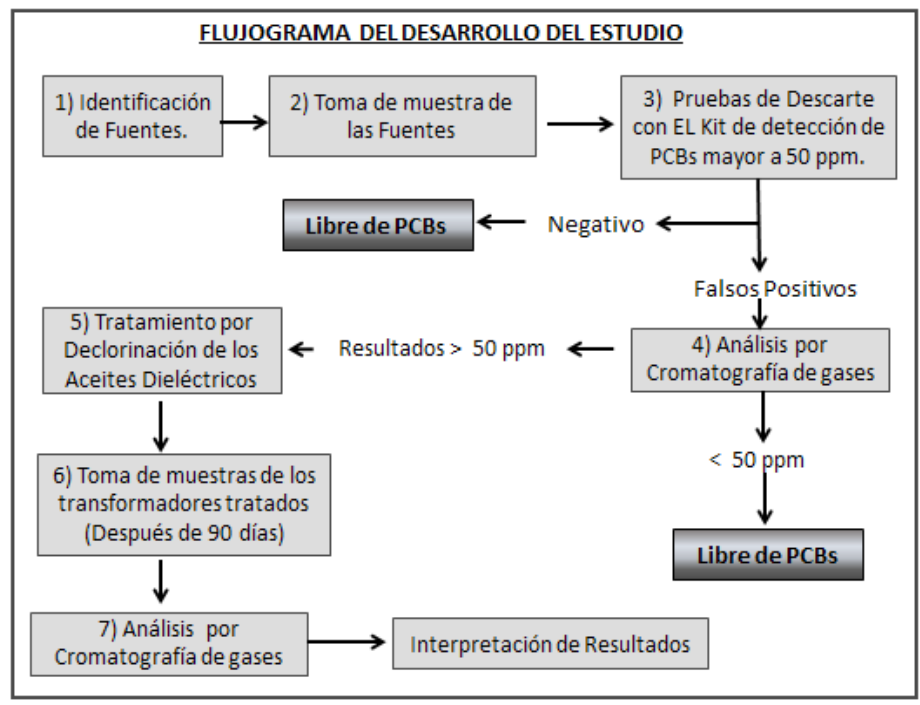

Figura 2. Flujograma del desarrollo de la investigación.

\section{Identificación de las fuentes}

Esta parte es el inicio del trabajo de investigación, se refiere a determinar todas las fuentes posibles donde se encuentran los PCBs en la unidad de estudio, los PCBs se encontraron únicamente en los transformadores antiguos de tensión, solicitando el listado de ubicación al área de mantenimiento eléctrico de la unidad de estudio, tanto de interior mina como de superficie, considerándose un total de 42 transformadores.

\section{Toma de muestras de aceites en las fuentes}

Teniendo el listado de ubicación de los transformadores se inició con la ubicación y toma de muestras de los aceites dieléctricos de cada transformador, teniendo las siguientes consideraciones:

Antes de iniciar con la toma de muestras, usar los equipos de protección personal como es: overol, casco, lentes y guantes de nitrilo, para evitar cualquier contacto directo con los PCBs.

Identificar en los transformadores, la válvula que presente la mejor condición para la toma de las muestras (por lo general en la parte inferior).

Colocar un recipiente o bandeja en la parte inferior de la válvula para prevenir contaminación al suelo en caso de derrames.

Abrir suavemente la válvula del transformador, colocar el frasco cerca a la boca de la válvula hasta llenar la cantidad de $(20 \mathrm{ml})$ y luego rotular los frascos con la ubicación correcta del transformador.

Al concluir la toma de muestras, dejar el lugar limpio y verificar el cierre correcto de las válvulas de los transformadores. 


\section{Prueba de descarte con el uso del kit de detección de PCBs Clor-N-Oil 50}

Una vez realizada la toma de muestras de los 42 transformadores, todos estos pasaron por la prueba de descarte, mediante el uso del kit Clor-N-Oil 50 de marca DEXSIL, para la detección de PCBs en los aceites dieléctricos a concentraciones mayores a 50 ppm, diseñado para ser utilizado en el campo, es rápido y fácil de utilizar. Cada caja contiene todo lo necesario para realizar la prueba en menos de 10 minutos. Todos los agentes reactantes están contenidos en ampollas con las cantidades precisas para obtener resultados al instante. En las figuras 3 y 4 , se muestra los resultados de las pruebas por el kit detector de PCBs Clor-N-Oil 50.

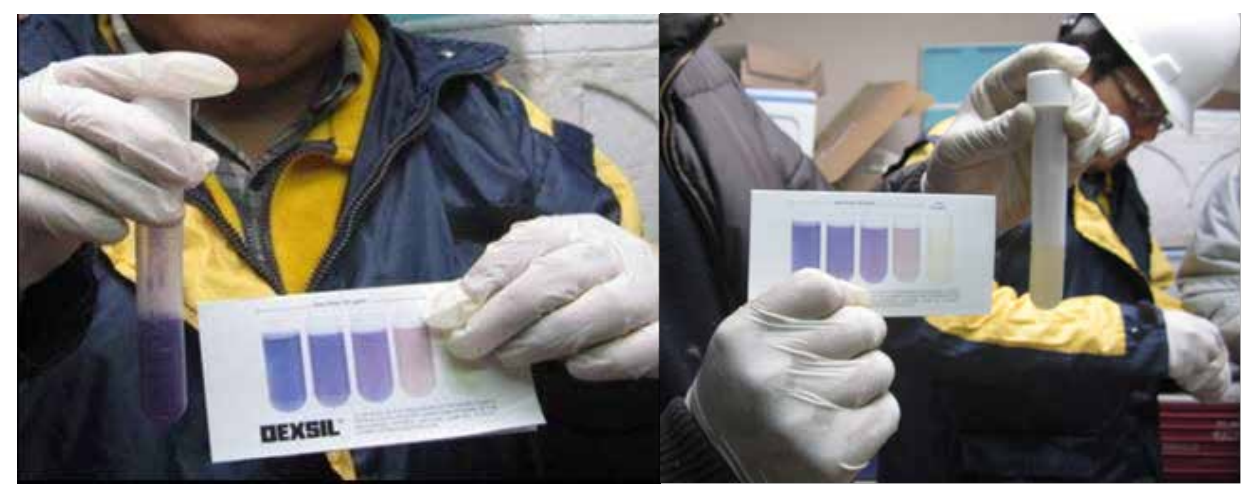

Figura 3. Resultados de las pruebas del kit colorimétrico; la vista del lado izquierdo, con resultado de color lila, representa aquellas muestras libres de PCBs y la vista del lado derecho del resultado de color crema amarillento, representa muestras con PCBs.

\section{Análisis por cromatografía de gases}

Luego de realizar el descarte con los kits de detección de PCBs a las 42 muestras de aceites, 13 muestras resultaron como falsos positivos y para conocer el contenido de concentración exacto de los PCBs, estas muestras se enviaron al laboratorio de la Dirección General de Saneamiento Ambiental (DIGESA), con acreditación del INDECOPI (0268.2014/SNAINDECOPI) y Acreditación del Instituto Nacional de Calidad (INACAL) con Registro $\mathrm{N}^{\circ}$ LE-080.

\section{Tratamiento por declorinación}

La tecnología desarrollada para el tratamiento de los PCBs fue impulsada por la empresa KIOSHI, una empresa Argentina, la cual trajo su tecnología al Perú, mediante la relación con el Ministerio del Ambiente, para ser aplicada en la unidad de estudio. El tratamiento de los aceites dieléctricos consta de dos partes; la primera y más importante es el proceso de la “declorinación” y la segunda es el proceso de "regeneración".

\section{Proceso de declorinación}

Este proceso se inicia retirando el aceite dieléctrico con PCBs de los transformadores, para llenar con aceite el reactor hasta la capacidad de 1500 litros, el reactor consta de dos tanques internamente; un tanque ubicado en la parte central del reactor, el cual posee una resistencia 
que aporta calor hacia la parte externa del tanque central, por esta razón el proceso controla dos registros de temperatura; tanto interna como externa del reactor, los cuales normalmente trabajan hasta $83{ }^{\circ} \mathrm{C}$, el reactor funciona en condiciones estandarizadas de presión y temperatura, una vez caliente el aceite pasa por la válvula de ingreso del vaso de soporte, en donde se ubica el sodio metálico, luego el aceite sale por la válvula de salida del vaso de soporte, regresando al reactor, hasta que se concluya el pase de todo el aceite. Al momento, cuando el aceite caliente entra en contacto con el sodio metálico, se genera la "reacción química de Wurtz", ya que este metal alcalino tiene fuerte afinidad con elementos halógenos, como es el cloro, descomponiendo los átomos de cloro presentes en las moléculas de los PCBs y generando nuevos compuestos orgánicos y dejando libre al cloro, en forma de cloruro inorgánico, el cual es completamente inerte y no posee propiedades nocivas, existiendo este en gran cantidad en la naturaleza ${ }^{14}$. En la figura 4 se observa la reacción de eliminación de los PCBs.

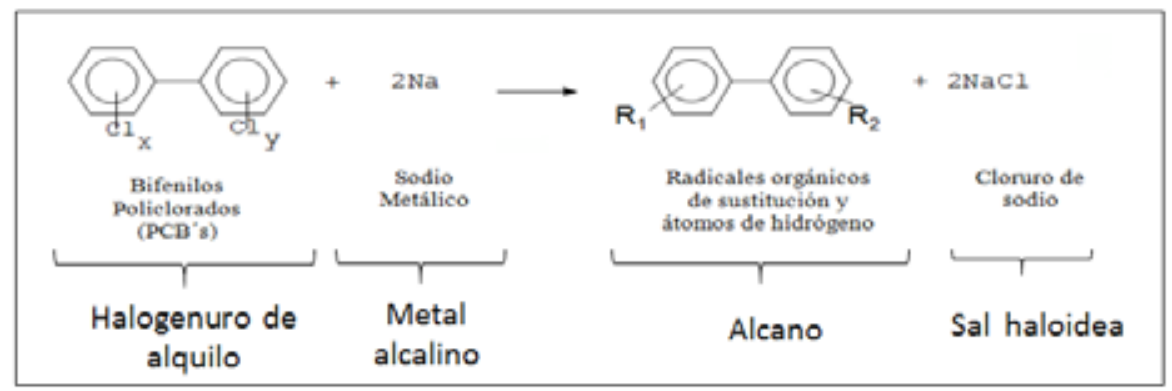

Figura 4. Reacción de eliminación de PCBs con sodio metálico.

Fuente: Kioshi (s.f.).

Una vez tratado el aceite, este se llena en las tolvas de decantación, sin embargo, el aceite tratado aún cuenta con impurezas (barro, sales inorgánicas, agua, vapores, etc), por eso se considera realizar el segundo proceso| de regeneración.

\section{Procesos de regeneración}

Este proceso se inicia pasando el aceite de las tolvas de decantación hacia el equipo de filtro prensa, para quitar las impurezas de barro y sales inorgánicas con el uso de tierras filtrantes, donde se optimizan las propiedades eléctricas del aceite, de tal forma recuperar las características aislantes del aceite, luego el aceite pasa a la secadora, ya que el aceite tratado puede contener un residual de humedad, el cual es eliminado en esta etapa por acción de la temperatura, porque la humedad afecta adversamente las características dieléctricas del aceite, concluyendo finalmente con el rellenado de los aceites tratados a los transformadores ${ }^{15}$.

En la figura 5 se muestra el proceso del tratamiento por declorinación y regeneración de los aceites dieléctricos contaminados con PCBs. 


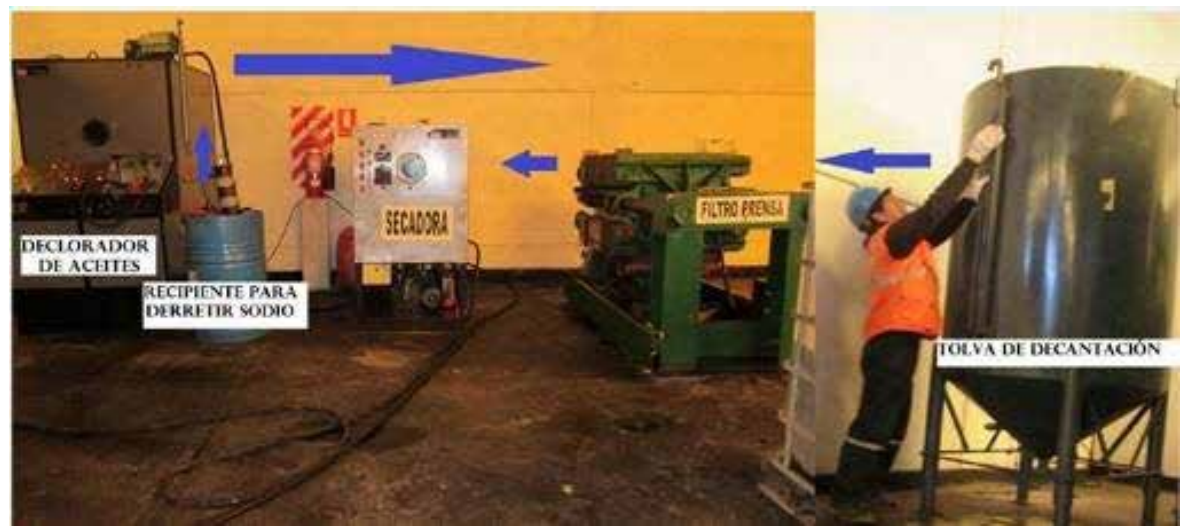

Figura 5. Proceso del tratamiento por declorinación y regeneración de PCBs, Inicia desde el equipo declorinador, pasa a las tolvas de decantación, secadora, filtro de prensa y rellenado del aceite.

\section{Toma de muestras y análisis por cromatografía de gases de aceites tratados}

Luego de ejecutado el tratamiento por declorinación de los aceites dieléctricos de los nueve transformadores, se volvió a realizar la toma de muestras de los aceites, después de los 90 días del tratamiento, para asegurar la descontaminación interna de los transformadores, las muestras también fueron enviadas al laboratorio de DIGESA para el análisis por cromatografía de gases.

\section{RESULTADOS Y DISCUSIÓN}

Cada secuencia del estudio de investigación tuvo resultados:

Primero, se inicio obteniendo la ubicación de las fuentes de PCBs en la unidad de estudio.

Segundo, se realizó la toma de muestras de los aceites, aquellas registradas en la lista de ubicación de la unidad de estudio, las cuales fueron 42 muestras registradas.

Tercero, obtenidas las muestras de los 42 transformadores, se realizó las pruebas de descarte con el uso de los kits detectores de PCB Clor-N-Oil 50, para identificar aquellos transformadores con resultado superior a los $50 \mathrm{ppm}$ de PCBs, y descartando aquellos transformadores sin PCBs, dando como resultado 13 muestras con resultados positivos, con esta prueba solo se llega a saber que se encuentran fuera del límite permitido, pero no se sabe con exactitud la cantidad exacta de PCBs, por esta razón las 13 muestras positivas pasaron por el análisis por cromatográfia de gases, para obtener resultados exactos.

Cuarto, de las 13 muestras analizadas por cromatografia de gases, solo 9 registraron resultados superiores a $50 \mathrm{ppm}$, los cuales fueron considerados para el tratamiento por declorinación. 
Quinto, los 9 transformadores con resultados mayor a $50 \mathrm{ppm}$, pasaron por el tratamiento por declorinación, que duró aproximadamente 30 días, tratando un total de 16250 litros de aceite, fue ejecutado por un profesional técnico perteneciente a la empresa Argentina KIOSHI, el cual se encargaba de todo el proceso del tratamiento por declorinación de PCBs.

Sexto, concluido el tratamiento por declorinación, se consideró esperar 90 días, esto por garantía de la empresa KIOSHI, después de concluido los 90 días se volvieron a realizar las tomas de muestras, y se enviaron al Laboratorio de DIGESA, para determinar si el tratamiento por declorinación es efectivo en cuanto a la reducción de los PCBs.

Sétimo, los resultados de las muestras de aceites tratados fueron enviados al laboratorio de DIGESA, donde se observan valores por debajo de los límites máximos permisibles $(<50$ ppm), esto quiere decir que el tratamiento por declorinación es muy eficiente, KIOSHI asegura que con este tipo de tratamiento se podría tratar aceites hasta con $10000 \mathrm{ppm}$ de PCBs.

En el cuadro 1, se muestran los resultados de laboratorio de DIGESA, donde se puede observar la diferencia de los resultados del antes y después del tratamiento por declorinación, observándose que los resultados de cuatro equipos se encuentran hasta por debajo del límite de detección del método de análisis que es de 1,6. En conclusión, todos los resultados después del tratamiento se observan por debajo de los $50 \mathrm{ppm} \mathrm{o} \mathrm{mg/L} \mathrm{de} \mathrm{PCBs,} \mathrm{que} \mathrm{es} \mathrm{el}$ límite máximo permisible de PCBs en aceites dieléctricos según la Environmental Protection Agency o Agencia de Protección Ambiental (EPA) de Estados Unidos, esto significando que mayor a $50 \mathrm{ppm}$ de $\mathrm{PCB}$, presenta un riesgo para la salud de los trabajadores y el ambiente.

Cuadro 1. Resultados de los análisis del antes y después del tratamiento de los aceites

\begin{tabular}{|c|c|c|c|c|c|c|}
\hline$N^{\circ} 1$ & Lugar & $\begin{array}{l}\text { Potencia } \\
\text { (KVA) }\end{array}$ & Marca & Nro. de Serie & $\begin{array}{c}\text { Antes } \\
\text { tratamiento } \\
\text { (ppm o } \mathrm{mg} / \mathrm{L} \text { ) }\end{array}$ & $\begin{array}{c}\text { Después } \\
\text { Tratamiento } \\
\text { (ppm/ mg/L) }\end{array}$ \\
\hline 1 & SE Atacocha & 500 & $\begin{array}{l}\text { General } \\
\text { Electric }\end{array}$ & Banco A 8638809 & 190,57 & 1,6 \\
\hline 2 & $\begin{array}{c}\text { SE Atacocha Nv } 4000 \\
\text { Serie B } 8638810\end{array}$ & 500 & $\begin{array}{l}\text { General } \\
\text { Electric }\end{array}$ & Banco A8638810 & 361,783 & 3,1 \\
\hline 3 & SE Atacocha Nv 4000 & 500 & $\begin{array}{l}\text { General } \\
\text { Electric }\end{array}$ & Banco A SN & 214,93 & 1,6 \\
\hline 4 & SE Chicrin & 561 & $\begin{array}{l}\text { General } \\
\text { Electric }\end{array}$ & F958033A & 570,47 & 1,6 \\
\hline 5 & SE Chicrin $8 \mathrm{~A}$ & 561 & $\begin{array}{l}\text { General } \\
\text { Electric }\end{array}$ & F958033B & 811,27 & 7,8 \\
\hline 6 & SE Chicrin 7A & 561 & $\begin{array}{l}\text { General } \\
\text { Electric }\end{array}$ & F958033C & 820,45 & 8,9 \\
\hline 7 & $\begin{array}{c}\text { SE Atacocha Nv } 4000 \\
N^{\circ} 8631051\end{array}$ & 500 & $\begin{array}{l}\text { General } \\
\text { Electric }\end{array}$ & Banco C 8631051 & 134,02 & 2,4 \\
\hline 8 & $\begin{array}{l}\text { SE Atacocha Nv } 4000 \\
N^{\circ} 8631052\end{array}$ & 500 & $\begin{array}{l}\text { General } \\
\text { Electric }\end{array}$ & Banco C 8631052 & 96,57 & 2,8 \\
\hline 9 & SE Chicrin 7B & 561 & $\begin{array}{l}\text { General } \\
\text { Electric }\end{array}$ & Banco C SN & 788,01 & 1,6 \\
\hline
\end{tabular}

Fuente: Resultado de los análisis por el laboratorio DIGESA, para la declaración de los PCBs de la unidad de estudio. 


\section{CONCLUSIONES}

- El desarrollo del tratamiento por declorinación demostró ser factible, redujo el contenido de PCBs en los aceites dieléctricos de los transformadores, la empresa KIOSHI asegura que mediante este método de tratamiento se pueden tratar los aceites que contengan hasta 10000 ppm de PCBs.

- El tratamiento por declorinación es un proceso químico, que ayuda a eliminar los átomos de cloro en los PCBs, ya que depende del grado de cloración los riesgos de tóxicidad y acumulación de los PCBs en las personas y en el medio ambiente.

- Este método de tratamiento por declorinación representa una alternativa de gestión para aquellas empresas usuarias de equipos electrónicos, los cuales contienen PCBs, o puedan iniciar con los inventarios y pruebas de descarte, ya que los kits detectores de PCBs Declor-N-Oil 50 no son costosos y se pueden encontrar en el Perú.

- De acuerdo al resultado final, se concluye que el tratamiento por declorinación elimina el riesgo de salud a los trabajadores y al medio ambiente, al reducir la concentración de los PCBs en los transformadores eléctricos de la unidad de estudio.

- Los ministerios de Salud y del Ambiente, deben fortalecer alternativas de solución para el manejo de los PCBs a corto plazo, fomentar empresas reconocidas que brinden el servicio de tratamiento de los PCBs con certificación y, además, fortalecer la normativa para su obligación a que todas las empresas de todos los sectores del país realicen la declaración de PCBs e implementen su gestión para su eliminación y se cuente con un registro digital del inventario actualizado; el cual, desde el año 2006, no cuenta con información actualizada a disposición del público, como sí lo desarrollan en otros países de América, como es el caso de Colombia, Chile, Brasil y México.

\section{AGRADECIMIENTO}

A la Universidad Nacional Mayor de San Marcos, a la Unidad de Posgrado de la Facultad de Ingeniería Geológica, Minera, Metalúrgica y Geográfica, por ofrecer las oportunidades de superación profesional.

A mi asesor, el Dr. Vidal Aramburú, por su apoyo y consejos en el desarrollo de esta investigación.

Al Ing. José Alcalá, por hacerme partícipe del proyecto del tratamiento por declorinación de bifenilos policlorados en la Cía Minera Atacocha SAA. 


\section{REFERENCIAS BIBLIOGRÁFICAS}

1. Suárez Montórfano R. Conociendo los bifenilos policlorados. Paraná, Argentina: Producciones educativas; 2006.

2. Astudillo Pillanga MA. Gestión de los transformadores eléctricos con bifenilos policlorados de la empresa Centrosur. [Tesis de posgrado]. Corrientes, Argentina: Universidad de la Cuenca del Plata; 2014.

3. Ospino I, Olivero J, Lans I, Vivas R. Modelo Qsar para la predicción de los tiempos de vida media de bifenilos policlorados en humanos. Vitae. 2005; 12(1): 45-53.

4. Crespo Ramírez M, Olano Goena I, Hernádez Jorgez E. Caso 2: Situación de la gestión de los PCB, estado de las tecnologías de descontaminación y destrucción sin incineración. Madrid: Paralelo Edición, SA; 2005

5. Salud Pública de México. PCB no obsoletos, un segundo vistazo a los subproductos de la fabricación de pigmentos. [Internet] México; 2012. [Citado 10 oct 2017] Obtenido de http://saludpublica.mx/index.php/spm/article/view/7218/9405.

6. Ministerio del Ambiente y Desarrollo Sostenible. (2015). Manual para la gestión integral de bifenilos policlorados - PCB. En Generalidades y conceptos básicos sobre bifenilos policlorados - PCB (pág. 8). Colombia. [Internet] Lima [Citado 23 set 2018] . Disponible en: HYPERLINK "http:/quimicos.minambiente.gov.co/images/quimicos/Tomos Manual/Tomo_1_generalidades_conceptos_pcb.pdf" http://quimicos.minambiente.gov. co/images/quimicos/Tomos_Manual/Tomo_1_generalidades_conceptos_pcb.pdf

7. Pfafflin J, Ziegler E. Effects of chimicals. En Enciclopedia of Enviromental Science and Engineering (pág 272). United Kingdom: Taylor and Francis Group; 2006

8. LaGreca M, Buckingham P, Evans J. Hazardous waste management. London, United Kingdoom: McGraw-Hill; 1996.

9. Centro Regional para América del Sur - CRBAS, Instituto Nacional de Tecnología Industrial (INTI), (2011). Herramientas para la toma de desiciones. En Gestión de PCB en la Industria Minera (pág. 19).

10. Wachong Solano LD. Análisis técnico de la implementación del proceso de declorinación, regeneración y recuperación del aceite dieléctrico contaminado con bifenilos policlorados. [Tesis de pregrado]. San José: Universidad de Costa Rica, Costa Rica; 2015

11. Ortiz A. Ministerio de Ambiente y Energía-Dirección General de Calidad Ambiental. Guía Técnica de PCBs en Costa Rica. San José, Costa Rica: Printea-Grupo Nación; 2015.

12. Aguirre J, Lopera E. Purificación de aceites aislantes contaminados con bifenilos policlorados (PCBs). Dyna. 2006; 150: 75-88.

13. Ministerio de Salud. Resolución Ministerial 683-2018. [Internet] Lima: Ministerio de Salud; 2018 [Citado 25 set 2018] . Disponible en: https://cdn.www.gob.pe/uploads/ document/file/176509/RM_0683-2018-MINSA.pdf

14. Kioshi. Proceso de Declorinación de Aceites Dieléctricos Contaminados con PCB. [Internet]. Buenos Aires:Kioshi [Citado 29 de set 2018). Disponible en:

15. Kioshi S.A. Regeneración de aceites dieléctricos de Transformadores. [DVD] Buenos Aires: Kioshi S.A.; 1998. 